\title{
HYBRID LANGUAGE MODELS AND SPONTANEOUS LEGAL DISCOURSE
}

\author{
P.E.Kenne and Mary O'Kane \\ The University of Adelaide \\ South Australia 5005 \\ Australia \\ $\{$ pek,mok\}@dvcr.adelaide.edu.au
}

\begin{abstract}
The idea of using multiple speech recognizers and alternate language models in a spoken language task is a familiar one. The problems which arise are deciding which language model(s) and/or recognizer(s) to use, or when to change language models. We use the idea of local perplexity of a corpus to determine when to change to an alternate language model and compare this strategy to a number of other strategies in the context of court interactions.
\end{abstract}

\section{INTRODUCTION}

The idea of using multiple speech recognizers and alternate language models in a spoken language task is a familiar one [1]. The problems which arise are deciding which language model(s) and/or recognizer(s) to use and when to change language models. For example, when working with recordings of court proceedings to automatically produce transcript, if a speaker change from a witness to a lawyer is detected (for exmaple, if lawyers and witnesses are recorded on separate tracks) it is simple to change from a language model for witnesses' speech to a language model for lawyers' speech.

We investigate this approach and several others.

\section{DATA}

The transcripts from two Australian court cases were used for training and testing. These transcripts do not include non-speech events such as "um" etc, but they do include repetitions, false starts etc. Table 1 gives details of training and test set sizes.

\begin{tabular}{|c|c|c|}
\hline & $\mathrm{c} 1$ & $\mathrm{c} 2$ \\
\hline Training size (words) & $155 \mathrm{~K}$ & $290 \mathrm{~K}$ \\
Test size (words) & $20 \mathrm{~K}$ & $40 \mathrm{~K}$ \\
\% Coverage & 88 & 92 \\
\hline
\end{tabular}

Table 1: Training/test set details

In case c1, the training set is the first ten days of a trial, and the test set is the next two days. In case $c 2$ the training set is the first twenty days of a trial and the test set is the next three days. Tables 2 and 3 give further details about each of the cases. In these tables we have combined training and test set details. Witnesses are assumed not to ask questions (not always true but the total number of questions asked by witnesses is very small). See Kenne et al [4] for further characterization of Australian court cases.

\begin{tabular}{|c|c|c|c|c|}
\hline & $\begin{array}{c}\text { Total } \\
\text { Words }\end{array}$ & $\begin{array}{c}\text { Total } \\
\text { Questions }\end{array}$ & $\begin{array}{c}\text { Total } \\
\text { Statements }\end{array}$ & $\begin{array}{c}\text { Average } \\
\text { utterance } \\
\text { length }\end{array}$ \\
\hline Judge1 & 17122 & 88 & 1027 & 15.3 \\
\hline Lawyers & 14699 & 614 & 122 & 19.9 \\
L1 & 10217 & 200 & 173 & 27.4 \\
L2 & 36805 & 1112 & 594 & 21.6 \\
L3 & 47921 & 1497 & 646 & 22.4 \\
L4 & 859 & 0 & 11 & 78.0 \\
L5 & 16 & 0 & 1 & 16.0 \\
L6 & & & & \\
Witnesses & & 0 & 11 & 19.5 \\
W1 & 215 & 0 & 4 & 19.0 \\
W2 & 76 & 0 & 169 & 10.2 \\
W3 & 1729 & 0 & 219 & 17.3 \\
W4 & 3798 & 0 & 540 & 10.8 \\
W5 & 5896 & 0 & 580 & 9.0 \\
W6 & 5197 & 0 & 51 & 10.8 \\
W7 & 550 & 0 & 168 & 11.0 \\
W8 & 1861 & 0 & 1821 & 13.4 \\
W9 & 24333 & 0 & & \\
\hline
\end{tabular}

Table 2: Details for case c1

\section{LANGUAGE MODELS AND LOCAL PERPLEXITY}

Several language model types were tested: word bigram, word trigram and word phrase bigram [5], all using a linear backing-off strategy [2]. For each model type and each case we used a model trained only on lawyers' speech, a model trained on both lawyers' and witnesses' speech and a model trained only on witnesses' speech. (Lawyers and wit- 


\begin{tabular}{|c|c|c|c|c|}
\hline & $\begin{array}{c}\text { Total } \\
\text { Words }\end{array}$ & $\begin{array}{c}\text { Total } \\
\text { Questions }\end{array}$ & $\begin{array}{c}\text { Total } \\
\text { Statements }\end{array}$ & $\begin{array}{c}\text { Average } \\
\text { utterance } \\
\text { length }\end{array}$ \\
\hline Judge2 & 8369 & 48 & 606 & 12.8 \\
\hline Lawyers & & & & \\
\hline $\mathrm{L} 7$ & 12924 & 362 & 142 & 25.6 \\
\hline L8 & 18186 & 507 & 257 & 23.8 \\
\hline L9 & 116920 & 4421 & 264 & 25.0 \\
\hline L10 & 52544 & 2214 & 132 & 22.4 \\
\hline Witnesses & & & & \\
\hline W10 & 383 & 0 & 83 & 4.6 \\
\hline W11 & 9366 & 0 & 555 & 16.9 \\
\hline W12 & 1457 & 0 & 124 & 11.8 \\
\hline W13 & 2290 & 0 & 245 & 9.4 \\
\hline W14 & 1152 & 0 & 95 & 12.1 \\
\hline W15 & 3352 & 0 & 130 & 25.8 \\
\hline W16 & 9395 & 0 & 682 & 13.8 \\
\hline W17 & 3906 & 0 & 266 & 14.7 \\
\hline W18 & 2563 & 0 & 109 & 14.7 \\
\hline W19 & 55851 & 0 & 3002 & 18.6 \\
\hline W20 & 887 & 0 & 71 & 12.5 \\
\hline W21 & 380 & 0 & 71 & 5.4 \\
\hline W22 & 549 & 0 & 44 & 12.5 \\
\hline W23 & 1901 & 0 & 153 & 12.4 \\
\hline W24 & 4733 & 0 & 339 & 14.0 \\
\hline W25 & 467 & 0 & 45 & 10.4 \\
\hline W26 & 6502 & 0 & 540 & 12.0 \\
\hline W27 & 15447 & 0 & 792 & 19.5 \\
\hline
\end{tabular}

For the court data sets, changing language models does not substantially change the shape of the local perplexity curve (see Kenne and O'Kane [3]). A different language model does however, translate the curve up or down.

\section{RESULTS}

Results from applying different language models to the test sets are given in tables 4-9.

Here, "Both" is the language model trained on all speakers, $\mathrm{L}+\mathrm{W}$ changes models from the lawyer model to the witness model when a change from lawyer to witness is detected and Hybrid uses local perplexity to change models. In the hybrid case the default action is to start with the model trained on all speakers, and if the local perplexity becomes sufficiently large (if the local perplexity exceeds the test set perplexity plus $12 \%$ ) a switch is made to the lawyer or witness language model as appropriate.

Observe that for case $c 2$ the perplexity and word error rate increase when using separate language models for lawyers and witnesses in some cases. This seems to be due to the fact that case $c 2$ has a number of expert witnesses giving evidence (W19 and W27) and their language is atypical of the other witnesses'. A possible solution for this difficulty is to use to lawyers' language model for such speakers.

There are several areas of further work: there is a (almost) closed set of lawyers who appear in cases in some areas of law and it would be of interest to develop language models for individuals. Similarly, although there is insufficient data to adequately model a judge's similar techniques may be applied. This would also allow techniques such as those described by Rudnikiy [6] to be used.

\begin{tabular}{|c|c|c|}
\hline Model & Word Error Rate & Effective Perplexity \\
\hline Both & 14.9 & 23 \\
L + W & 12.2 & 20 \\
Hybrid & 11.7 & 19 \\
\hline
\end{tabular}

Table 4: Results for case c1: word bigram

the perplexity $P P$ of this corpus with respect to the language model is defined as

$$
P P=\exp \left(-\frac{1}{N} \operatorname{Pr}\left(w_{1} w_{2} \ldots w_{N}\right)\right)
$$

The perplexity may thought of as approximately the average branching factor in the language model. Most of the literature reports a single value of $P P$ for a language model/corpus combination. However, there is no reason why a number of values of perplexity (corresponding to different parts of the corpus) may not be calculated.

Figure 1 shows the local perplexity (for a word bigram model) for the start of case $c 2$ test set, using fixed windows of 600 and 1200 words, with $90 \%$ overlap in both cases. The perplexity of this data is 42 .

Local perplexity was calculated for the remainder of these experiments by using fixed size of 600 words and $90 \%$ overlap.

\begin{tabular}{|c|c|c|}
\hline Model & Word Error Rate & Effective Perplexity \\
\hline Both & 10.8 & 42 \\
L+W & 11.2 & 36 \\
Hybrid & 9.8 & 34 \\
\hline
\end{tabular}

Table 5: Results for case c2:word bigram

\section{REFERENCES}

1. Chevalier, H., Ingold, C., Kunz, C., Moore, C., Roven, R., Yamron, J., Baker, B., Bamberg, P., Bridle, S., Bruce, T., and Weader, A. Large-vocabulary speech recognition in specialized domains. In Proceedings ICASSP 95, pages 217-220, May 1995. 


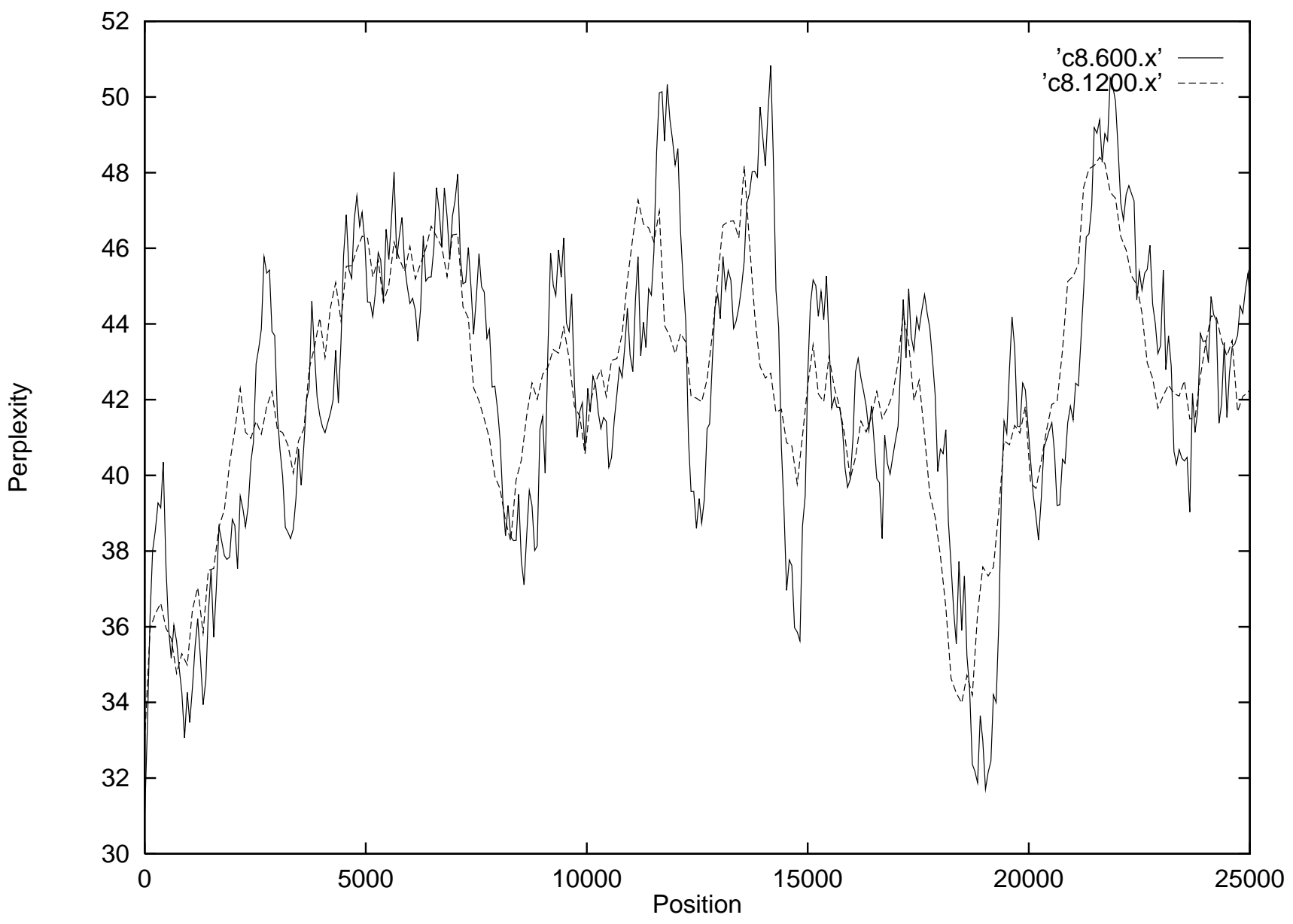

Figure 1: Local perplexity for case c2 for windows of size 600 and 1200

\begin{tabular}{|c|c|c|}
\hline Model & Word Error Rate & Effective Perplexity \\
\hline Both & 9.7 & 13 \\
L + W & 9.2 & 11 \\
Hybrid & 9.2 & 10 \\
\hline
\end{tabular}

Table 6: Results for case c1: word trigram

\begin{tabular}{|c|c|c|}
\hline Model & Word Error Rate & Effective Perplexity \\
\hline Both & 11.9 & 28 \\
L + W & 12.4 & 29 \\
Hybrid & 9.8 & 26 \\
\hline
\end{tabular}

Table 7: Results for case c2:word trigram

2. Ney H., Essen U., and R. Kneser. On structuring probabalistic dependences in stochastic language modelling. Computer Speech and Language, 8:1-38, 1994.

3. Kenne P.E. and O'Kane M. Topic change and local perplexity in spoken legal dialogue. In Proceedings of the International Conference on Spoken Language Processing

\begin{tabular}{|c|c|c|}
\hline Model & Word Error Rate & Effective Perplexity \\
\hline Both & 11.8 & 16 \\
L + W & 11.8 & 14 \\
Hybrid & 10.9 & 13 \\
\hline
\end{tabular}

Table 8: Results for case c1: word phrase bigram

\begin{tabular}{|c|c|c|}
\hline Model & Word Error Rate & Effective Perplexity \\
\hline Both & 12.4 & 36 \\
L $+\mathrm{W}$ & 12.3 & 36 \\
Hybrid & 12.2 & 34 \\
\hline
\end{tabular}

Table 9: Results for case c2:word phrase bigram

ICSLP96, October 1996.

4. Kenne P.E., O’Kane M., and Pearcy H.G. An australian speech database derived from court recordings. In Proceedings of the Fifth Australian International Conference on Speech Science and Technology, pages 808-813, December 1994. 
5. Kenne P.E., O’Kane M., and Pearcy H.G. Language modeling of spontaneous speech in a court context. In Proceedings Eurospeech 95, pages 1801-1804, September 1995.

6. A.I. Rudnicky. Language modeling with limited domain data. In Proceedings of the Spoken Language Systems Technology Workshop, pages 66-69, January 1995. 